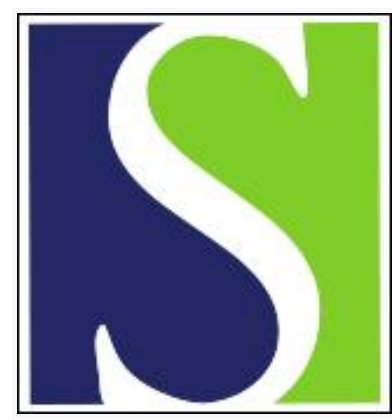

Scand J Work Environ Health 2008;34(2):151-157

https://doi.org/10.5271/sjweh.1219

Published online: 12 May 2008, Issue date: 31 Apr 2008

High risk for nasal carriage of methicillin-resistant Staphylococcus aureus among Danish veterinary practitioners by Moodley A, Nightingale EC, Stegger M, Nielsen SS, Skov RL, Guardabassi L

Affiliation: Department of Veterinary Pathobiology, Stigbøjlen 4, DK-1870 Frederiksberg C, Denmark. asm@life.ku.dk

The following article refers to this text: 2010;36(6):515-516

Key terms: Staphylococcus aureus; antibiotic resistance; Denmark; farmer; methicillin resistancy; nasal carriage; occupational health; risk; veterinarian; veterinary practitioner

This article in PubMed: www.ncbi.nlm.nih.gov/pubmed/18470441 


\title{
High risk for nasal carriage of methicillin-resistant Staphylococcus aureus among Danish veterinary practitioners
}

\author{
by Arshnee Moodley, MSc (Med), ${ }^{1}$ Emily C Nightingale, DVM, ${ }^{1}$ Marc Stegger, MSc, ${ }^{2}$ Søren S Nielsen, \\ PhD, ${ }^{3}$ Robert L Skov, MD, ${ }^{2}$ Luca Guardabassi, PhD'
}

\begin{abstract}
Moodley A, Nightingale EC, Stegger M, Nielsen SS, Skov RL, Guardabassi L. High risk for nasal carriage of methicillin-resistant Staphylococcus aureus among Danish veterinary practitioners. Scand J Work Environ Health. 2008;34(2):151-157.
\end{abstract}

Objectives Due to their multiple antibiotic resistance properties, methicillin-resistant Staphylococcus aureus (MRSA) are a major public health problem. After the recently described emergence of MRSA in animals, the authors investigated the risk of nasal MRSA carriage among people with professional contact with animals.

Methods Nasal swabs and information on animal exposure and known MRSA risk factors were obtained from participants $(\mathrm{N}=702)$ at five conferences organized by national veterinary and farmer associations and students at a business school in Denmark. All of the participants were screened by standard microbiological techniques for MRSA detection and characterization.

Results MRSA carriage was significantly $(\mathrm{P}<0.02)$ higher among the veterinary practitioners $(3.9 \%)$ than among the participants not professionally exposed to animals $(0.7 \%)$. Six of the nine MRSA strains isolated from veterinary practitioners belonged to clonal complexes (CC) previously associated with horses (CC8), small animals (CC22), and pigs (CC398). Although four of the nine positive veterinarians carried the CC associated with pigs, exposure to small animals, cattle, or horses, but not to pigs, was found to be a significant risk factor. Conclusions The results indicate that veterinarians are at risk of MRSA carriage. Veterinary professionals should be informed about this emerging occupational health risk and educated about preventive measures. Collaboration between national medical and veterinary institutions is urgently needed to control the spread of these unwanted bacteria in the community.

Key terms antibiotic resistance; farmer; occupational health; veterinarian.

Staphylococcus aureus form one of the most important bacterial pathogens worldwide. Methicillin-resistant $S$ aureus (MRSA) are a common cause of hospital-acquired infections that are associated with high morbidity and high mortality (1-3). MRSA are often resistant to most of the antibiotics available for treating staphylococcal infections, and they represent an economic burden on society due to increased therapeutic and diagnostics costs and the need for prolonged hospitalization. Recently, an increasing number of reports has documented cases of MRSA colonization or infection in animals, namely, dogs, cats, horses, and pigs (4-7). As in humans, MRSA can colonize the skin and mucosa of healthy animals, especially nasal mucosa, which is used as the standard sampling site for surveys on human or animal carriage. MRSA were initially reported in pets owned by human patients infected with these bacteria (8) or by people having known risk factors for MRSA colonization, such as hospital employees (9). Thus it was hypothesized that MRSA in animals were mainly transmitted from humans. However, transmission can potentially occur in both directions, and direct exposure to MRSA-positive animals can lead to transmission to humans and potential infection. Various studies have

1 Department of Veterinary Pathobiology, Faculty of Life Sciences, University of Copenhagen, Frederiksberg C, Denmark.

2 Staphylococcal Reference Laboratory, Statens Serum Institut, Copenhagen, Denmark.

3 Department of Large Animal Sciences, Faculty of Life Sciences, University of Copenhagen, Frederiksberg C, Denmark.

Correspondence to: Arshnee Moodley, Department of Veterinary Pathobiology, Stigbøjlen 4, DK-1870 Frederiksberg C, Denmark. [E-mail: asm@life.ku.dk] 
recently demonstrated the transmission of MRSA between animals and people in daily contact with animals, such as veterinarians (10-12) and pig farmers (7).

To investigate possible occupational risks associated with the emergence of MRSA in animals, three independent studies were carried out to assess the prevalence of MRSA nasal carriage in veterinary personnel attending conferences [ie, the American College of Veterinary Internal Medicine (ACVIM) Forum in Louisville, Kentucky, USA (13), the International Pig Veterinary Society Congress (IPVS) in Copenhagen, Denmark (14), and a national livestock conference in the Netherlands (15). The proportion of nasal MRSA carriage observed in the three studies was high among the veterinary personnel $(7.1 \%, 12.5 \%$, and $3.9 \%$, respectively) when compared with the estimated community prevalence $(0.3 \%$ to $0.8 \%)(16,17)$. At the ACVIM Forum, MRSA carriers were more frequently detected among technical staff $(14.7 \%)$ and large animal personnel (15.6\%) than among small animal personnel (4.4\%) (13). Similarly, at the IPVS, $94 \%$ of the MRSA-positive persons had frequent contact with pigs, whereas only $47 \%$ of the MRSA-negative persons had frequent contact with pigs. This finding suggests that exposure to these animals is a risk factor for MRSA carriage (14). All strains isolated at the Dutch national livestock conference (15) and 31 out of 34 strains from the IVPS (14) belonged to clonal complex (CC) 398, the MRSA lineage associated with pigs.

The incidence of hospital-acquired MRSA has been maintained at low levels in Denmark since the mid1970s, probably as a result of the national control program based on increased hospital hygiene awareness, the implementation of strict infection control practices, and prudent antibiotic consumption. Up until 2002, the annual incidence of MRSA infections was $\leq 1 \%$ of total $S$ aureus isolates from blood. Since then, an apparent increase has been observed, up to approximately $2 \%$ in 2005 (18). In view of the recent increase in the incidence of MRSA infections, since November 2006, MRSA infection and carriage have become notifiable (ie, all MRSA cases have to be reported to the national health authorities). However, Denmark remains a country with one of the lowest MRSA infection prevalences in $\mathrm{Eu}$ rope. Among animals, only one case of MRSA carriage has been reported to date (19).

The three previous studies on MRSA carriage among veterinary staff lacked control groups of people without professional contact with animals and had relatively low sample sizes (179 to 272 persons tested), and two of the studies included heterogeneous study populations consisting of veterinarians from many different countries. The objective of our cross-sectional study was to determine whether people with different forms of professional contact with animals are at risk of MRSA carriage in Denmark, a country where MRSA are uncommon in both the human and animal populations. For this purpose, standard microbiological techniques were used to screen approximately one-tenth $(\mathrm{N}=231)$ of the reference population of veterinary practitioners in Denmark for MRSA carriage. The survey also included cattle farmers, other professionals exposed to animals, and a control group of people without professional contact with animals, for a total of 725 tested persons.

\section{Study population and methods}

\section{Study population}

The sampling was performed between August 2006 and February 2007 at the following five national conferences attended mostly by veterinary staff or farmers: (i) the Annual Veterinary Professional Week, Nyborg; (ii) a meeting of the Danish Pig Veterinary Society, Kolding; (iii) a general meeting of the Danish Equine Veterinary Association, Ebeltoft; (iv) a general meeting of the Danish Small Animal Veterinary Association, Århus; and (v) the Danish cattle conference, Herning. The study population comprised the following four categories: (i) veterinary practitioners in contact with production (eg, pigs and cattle) or companion animals (eg, horses, dogs, and cats), (ii) cattle farmers, (iii) other nonveterinary professions with daily contact with animals (eg, veterinary or agricultural students or veterinary nurses), and (iv) unexposed persons (ie, persons without professional exposure to animals). Students from the Copenhagen Business School were also recruited for their lack of professional contact with animals.

\section{Sampling and questionnaire information}

The participants were provided with a single cotton swab and instructed to insert the cotton swab $\sim \mathrm{cm}$ into each nostril and rotate along the mucosal membrane for 5 seconds. Sampling was performed under the supervision of one member of the scientific staff to ensure that the swabs were taken correctly. Information about the amount of weekly professional contact with specific animal species and information on recognized risk factors for MRSA carriage, such as previous hospitalization (within the last 6 months), antimicrobial therapy (within the last 6 months), and residence with a human health care worker, was obtained using a standardized questionnaire filled out prior to the sampling. Participation was on a strict voluntary basis, and signed informed consent was obtained from all of the participants. Personal information was kept anonymous except for the volunteers from the Danish cattle conference, who were sampled after 1 November 2006 when MRSA became 
mandatorily reportable in Denmark. The protocols used were approved by the Danish National Committee on Biomedical Research Ethics (H-KF-2007-0007).

\section{Bacterial identification and characterization}

MRSA were detected by the following two methods: (i) direct streaking onto oxacillin resistance screening agar (ORSAB, Oxoid, Hampshire, United Kingdom) and (ii) after streaking onto ORSAB swabs were placed into a MRSA-selective enrichment broth (Statens Serum Institut, Copenhagen, Denmark), followed by streaking on MRSA-ID agar plates (BioMérieux, Marcy l'Etoile, France). After 24 and 48 hours of incubation at $37^{\circ} \mathrm{C}$, presumptive MRSA colonies on either of the chromogenic agar plates were subcultured onto $5 \%$ blood agar plates and a 1- $\mu \mathrm{g}$ oxacillin disc (Oxoid). MRSA isolates were confirmed through the detection of the gene responsible for methicillin resistance (mecA) by polymerase chain reaction (PCR) (20) and by PCR detection and sequencing of the staphylococcal protein A ( $s p a)$ gene (21). The sequences were analyzed using Ridom StaphType 1.5.2 software and submitted to the Ridom spa server database (http://spa.ridom.de/spatypes.shtml, last accessed on 11 May 2007) to determine possible associations with $\mathrm{CC}$ identified by multi-locus sequence typing. SCCmec types were determined with a multiplex PCR as described by Oliveira et al (22). The presence of the Panton-Valentine leukocidin (PVL) toxin gene was determined by PCR as described by Lina et al (23).

\section{Antimicrobial susceptibility}

Antimicrobial susceptibility testing was performed by the disk diffusion method of the Clinical and Laboratory Standards Institute (24), unless stated otherwise. The following antimicrobial disks (Oxoid) were used: chloramphenicol (30 mg), ciprofloxacin (5 mg), clindamycin (2 mg), erythromycin (15 mg), fusidic acid (10 $\mathrm{mg}$ ), kanamycin (30 mg), mupirocin (5 mg), quinupristin or dalfopristin $(15 \mathrm{mg})$, rifampicin $(5 \mathrm{mg})$, tetracycline (30 mg), and trimethoprim and sulfamethoxazole (1.25 $+23.75 \mathrm{mg}$ ). Disc diffusion susceptibility was tested for fusidic acid and mupirocin as previously described $(25,26)$. The reference strain S aureus ATCC 25923 was used for quality control.

\section{Statistical analysis}

Because of the low number of MRSA-positive isolates in most of the groups, the association between the occurrences of MRSA between each hypothesized risk factor was assessed with the use of the exact logistic analysis with the statistical package LogExact 4 (27). Odds ratios were estimated from the following general formula on the basis of the estimates of the logistic analysis as follows: $\ln \left(P_{i} /\left(1-P_{i}\right)\right)=B_{i}$, where $P_{i}=\operatorname{Pr}$ (MRSA + risk factor $i$ ) and $B_{i}$ is the effect of the $i$ th risk factor group. The difference in the prevalence of MRSA between the risk factor groups was tested using the exact test. Conditional maximum likelihood estimates were calculated. If the observed value was at the extreme (eg, zero positive persons in a group), the conditional maximum likelihood estimate did not exist and was replaced by the median unbiased estimate.

The numbers of hours spent with animals were grouped as follows: (i) 0 hours/week, (ii) $>0$ and $\leq 5$ hours/week, (iii) $>5$ and $\leq 20$ hours/week, and (iv) $>21$ hours/week. These time groups were stratified by animal species (small animals, pigs, cattle, horses, and other) and also considered as a combined group.

\section{Results}

Questionnaires and samples were obtained from 725 persons. Altogether 23 observations were excluded from the data analyses for the following reasons: 20 samples were from foreigners (three from Finland, one from France, one from Germany, five from Norway, seven from Sweden, one from Switzerland, one from the Netherlands, and one from the United Kingdom) and therefore did not represent the target population; one was from a human health care nurse and therefore constituted a separate risk group; two persons did not fill in relevant information in the questionnaire. All the samples from these 23 persons were MRSA-negative. The remaining 702 persons comprised veterinary practitioners $(\mathrm{N}=231)$, nonveterinarians $(\mathrm{N}=72)$, farmers $(\mathrm{N}=98)$, and unexposed persons $(\mathrm{N}=301)$.

The distribution of MRSA carriers from each of the sampling events is given in table 1 .

Table 1. Distribution of methicillin-resistant Staphylococcus aureus (MRSA) obtained during six distinct sampling events.

\begin{tabular}{lrrr}
\hline & \multicolumn{2}{c}{ MRSA } & \multirow{2}{*}{ Total } \\
\cline { 2 - 3 } & \multicolumn{2}{c}{ Positive } & Negative \\
\cline { 2 - 3 } & & & \\
Danish Veterinary Association-horse section, & 1 & 48 & 49 \\
Ebeltoft & - & 169 & 169 \\
Danish cattle conference, Herning & 2 & 65 & 67 \\
Danish Pig Veterinary Society, Kolding & 5 & 185 & 190 \\
Annual Veterinary Professional Week, Nyborg & & 96 & 99 \\
Danish Small Animal Veterinary Association, & 3 & 96 & 128 \\
Århus & - & 128 & \\
Copenhagen Business School & 11 & 691 & 702 \\
\hline Total & &
\end{tabular}


MRSA was isolated from 11 persons: $3.9 \%(\mathrm{~N}=9)$ of veterinary practitioners and $0.7 \%(\mathrm{~N}=2)$ of the unexposed group. The veterinary practitioners had a significantly $(\mathrm{P}=0.02)$ higher odds of carrying MRSA than did the practitioners in the unexposed group (table 2). The chance of carrying MRSA was not higher among the farmers and other persons with professional contact with animals than among the unexposed group.

Time spent with animals was a significant risk factor only for people spending 5 to 20 hours/week with small animals $(\mathrm{P}=0.02)$, cattle $(\mathrm{P}<0.001)$, or horses $(\mathrm{P}<0.001)$, but not pigs $(\mathrm{P}=0.86)$. This time frame was typical for veterinary practitioners, but, because of the sparse data material, it could not be determined whether this association was due to the number of hours spent with the animals or to other factors associated with their profession. Not all of the veterinarians stated whether they used gloves while handling antibiotics. Therefore, it was not possible to determine if the handling of antibiotics without gloves was a risk factor for MRSA carriage. Of the veterinary practitioners that answered this question

Table 2. Distribution of methicillin-resistant Staphylococcus aureus (MRSA) in different occupational groups. (OR = odds ratio, $95 \% \mathrm{Cl}=95 \%$ confidence interval)

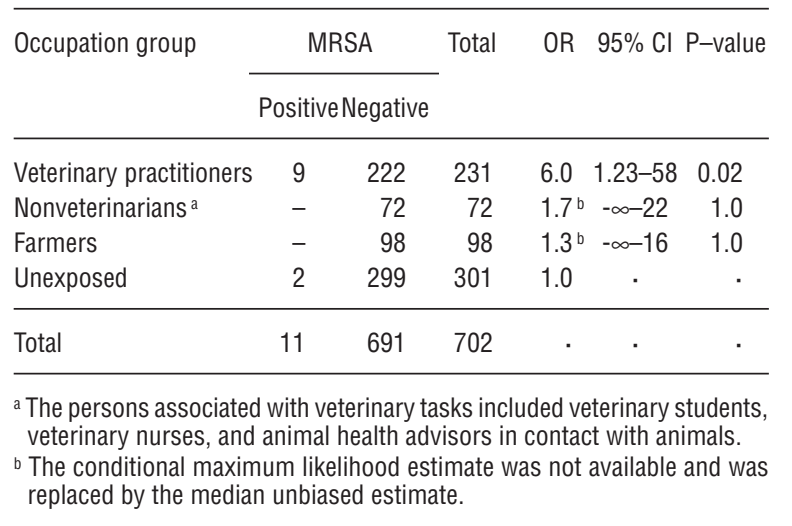

(149 of 231), 135 stated that they did handle antibiotics without using protective gear. Four of the five MRSApositive veterinarians stated that they handled antibiotics without gloves. There was no apparent risks associated with visits to the hospital, the occupation of the spouse, the personal consumption of antibiotics within the past 6 months, and the presence of pets in the household (data not shown).

All of the MRSA isolates were negative for the $p v l$ toxin gene. Of the 11 strains, 5 carried SCCmec type IV cassettes, and the remaining isolates were nontypeable by the Oliveira method, including all of the CC398 isolates. Nine MRSA strains were resistant to erythromycin, and all of the strains belonging to CC398 were resistant to tetracycline (table 3). Sequencing of the protein A gene (spa) revealed high genetic diversity (table 3), including spa types associated with CC8 (t008), CC22 (t020 and t022), and CC398 (t011 and t034).

\section{Discussion}

This study confirmed that veterinary staff form a professional category at risk of MRSA carriage in that the veterinarians in our study had a higher MRSA prevalence $(3.9 \%)$ than the members of the unexposed group $(0.7 \%)$. The MRSA carriage rate observed for the veterinary staff was also high when compared with the expected prevalence among healthy persons in the Danish community, which is well below $1 \%$. In particular, practicing veterinarians with 5 to 20 hours of professional contact with small animals, cattle, and horses were shown to have a higher chance of being MRSA carriers than the other groups. In contrast with the findings of previous studies, no statistically significant association could be shown between MRSA carriage and contact with pigs.

Table 3. Description of the methicillin-resistant Staphylococcus aureus carriers and strains. $(\mathrm{CHL}=$ chloramphenicol, $\mathrm{CIP}=$ ciprofloxacin, $\mathrm{CLI}=$ clindamycin, ERY = erythromycin, FD = fusidic acid, $K A N=$ kanamycin, MUP = mupirocin, $\mathrm{Q}-\mathrm{D}=$ quinupristin/dalfopristin, TET = tetracycline, SXT = trimethoprim/sulfamethoxazole, NA = not applicable)

\begin{tabular}{|c|c|c|c|c|c|}
\hline Risk group & Type of practice & spa type & Clonal complex & SCCmec type & Antibiotic resistance profile \\
\hline Veterinarian & Horse & t166 & CC1 & NT & ERY, KAN, SXT, TET \\
\hline Veterinarian & Cattle, horse, small animal & t008 & CC8 & IV & CIP, CLI, ERY, KAN \\
\hline Veterinarian & Cattle, horse, small animal & t020 & CC22 & IV & CIP, CLI, ERY \\
\hline Veterinarian & Cattle, horse, small animal & t022 & CC22 & IV & CIP \\
\hline Veterinarian & Cattle & t216 & CC59 & IV & CIP, CLI, ERY \\
\hline Veterinarian & Cattle, small animal & t186 & CC88 & NT & CIP, ERY, TET \\
\hline Veterinarian & Small animal & t034 & CC398 & NT & CHL, CLI, ERY, FD, MUP, SXT, TET \\
\hline Veterinarian & Pig & t034 & CC398 & NT & TET \\
\hline Veterinarian & Small animal & t034 & CC398 & NT & ERY, TET \\
\hline Unexposed & NA & t011 & CC398 & NT & CHL, CLI, ERY, FD, SXT, TET \\
\hline Unexposed & NA & t002 & CC5 & IV & CLI, ERY, KAN, Q-D \\
\hline
\end{tabular}


This discrepancy could reflect geographic differences in the prevalence of MRSA in pigs. A recent survey in the Netherlands (28) showed a high prevalence (39\%) among pigs in that country. However, the prevalence of MRSA in Danish pigs is likely to be substantially lower $(19,29)$, and this lower prevalence may explain the rare occurrence of these bacteria in Danish pig practitioners. Although one of the previous surveys of MRSA carriage in veterinary staff took place at a veterinary conference (IPVS) in Denmark (14), MRSA were detected in only one Danish pig practitioner despite the high prevalence of MRSA observed among pig practitioners from other countries attending the conference (14).

Interestingly, cattle farmers were not found to be a risk group, and none of the cattle farmers tested were found to carry MRSA. This result suggests that the risk of MRSA carriage may differ between distinct professional groups working in contact with the same animal species. It should be noted that veterinary practitioners generally work with a variety of different animal species and are exposed to many animal populations, whereas farmers are in contact with one or a few animal species and populations. Therefore, the risk of MRSA carriage may be related to the type of professional contact.

Although most of the recorded factors were considered risk factors, the observed low prevalence of MRSA made inference with respect to these factors difficult. Exact logistic analyses were used to derive odds ratios and $95 \%$ confidence intervals. However, conditional maximum likelihood estimates were not always available and were therefore replaced by median unbiased estimates. The point estimates of the latter should be interpreted with care, and more weight should be put on the confidence intervals. In general, a low prevalence in the various risk groups constitutes a special problem that renders studies of risk factors difficult. By the use of exact methods, it is possible to some extent to circumvent some limitations concerning sample size, but a larger sample size would still be required for appropriate inferences with respect to some risk factors. It should be noted that the sample size of this study was approximately 2.5 times larger than those used in the three previous surveys. Furthermore, this survey was conducted at the national level and therefore targeted a smaller reference population than those of two previous studies performed at international veterinary conferences. Denmark is a small country with approximately 747 practicing veterinarians registered with the national veterinary association, including 439 small animal, 265 cattle, 149 pig, and 192 horse practitioners (it should be noted that a veterinary practitioner may be a member of more than one section at a time) (Charlotte Borelli, Danish Veterinary Association, personal communication, 2007). Thus the sample size of our study was relatively high (approximately one-third of the total target popula- tion) when the low number of practicing veterinarians in this country is taken into consideration.

Six of the nine MRSA isolated from veterinary practitioners belonged to $\mathrm{CC}$ groups $(\mathrm{CC} 8, \mathrm{CC} 22$, and CC398) that have previously been associated with horses, small animals, and pigs, respectively (10, 30-32). In a previous study (12), it was shown that MRSA isolated from small animal practitioners in the United Kingdom and Ireland belonged to the same genetic lineage as MRSA occurring in small animals, whereas MRSA isolated from horse practitioners were genetically related to strains occurring in horses. These associations were considered to be indirect evidence for the zoonotic transmission of MRSA from animals to veterinary staff. The CC occurring in small animals and horses in Europe (CC22 and CC8, respectively) are established human pathogens, and they probably originated from humans. The genetic lineage that recently emerged in pigs (CC398) is likely to be of animal origin and may be regarded as true zoonosis. Several cases of human infections associated with this zoonotic MRSA lineage (including severe infections) have been reported in the Netherlands (32), Germany and Austria (33), and, more recently, in Denmark (Robert Skov, unpublished results).

Four veterinarians carried the pig-associated clone CC398. In concordance with the results of previous studies in the Netherlands $(7,15)$, in our study, CC398 isolates were resistant to tetracycline, the antibiotic most frequently used in pig farming. However, all four CC398 isolates had very heterogeneous resistance patterns, even within the same spa type (table 3). Furthermore, only one of the four people harboring MRSA CC398 (three veterinary practitioners and one unexposed person) noted contact with pigs ( 25 hours/week). The remaining two veterinary practitioners had 37 hours/week with small animals, a finding suggesting that CC398 may be linked to animals other than pigs. A previous study by Witte et al (33) provided a similar indication, as MRSA strains belonging to CC398 were isolated from cases of sinusitis and wound infections in dogs and horses. The unexposed CC398 carrier was a sales representative at a pharmaceutical company, who was likely to have had frequent contact with veterinary practitioners. The remaining MRSA isolates belonged to $\mathrm{CC}$ groups previously identified in Denmark and known to cause community-acquired infections. The occurrences of MRSA belonging to these $\mathrm{CC}$ groups have not been reported in animals to date.

Factors other than exposure to animals should be considered as possible risk factors. For example, handling antimicrobials without gloves might be an alternative risk factor. $S$ aureus is part of the normal skin flora, and repeated exposure to antimicrobials could lead to selection for MRSA. In this study, it was not possible to determine whether the use of protective gear during 
the handling of antibiotics was associated with MRSA carriage. However, handling antimicrobials without gloves seemed to be a common practice among Danish veterinarians and, more importantly, among most of those who were found to carry MRSA. Veterinary professionals should be informed about this emerging occupational health risk and educated about preventive measures. For example, they should wear gloves when handling antimicrobial agents and take adequate protective measures when attending animals at risk of MRSA infection (eg, wound and postoperative infections) and working in farm environments, which can be contaminated with MRSA (eg, pig farms). Skin lesions should always be protected from exposure to domestic animals, as indicated by recent documentation on skin MRSA infections transmitted from horse to veterinary personnel (34). The risk of zoonotic MRSA infection can be minimized by the early recognition of infected animals, proper animal handling, biosecurity precautions, and, most importantly, personal hygiene.

This study indicates that, even in a country such as Denmark, where MRSA are rare in both human and animal populations, the prevalence of MRSA carriage among veterinary practitioners is significantly higher than in the rest of the community. This finding raises important questions related to both occupational and public health concerns. Animals can act as reservoirs of novel MRSA lineages, and veterinarians should be aware of the importance of MRSA in animals and should be able to detect and manage animal patients, as well as to prevent the spread of MRSA within the animal population. Comprehensive guidelines on how to manage MRSA in veterinary practices have recently been developed by the British Small Animal Veterinary Association (BSAVA) and are available on the Internet (http://www.bsava.com/resources/mrsa/mrsaguidelines/ mrsaguidelines.htm).

In summary, veterinary practitioners spending between 5 to 20 hours a week had a higher chance of being MRSA-positive than an unexposed group. In view of this finding, increased awareness, appropriate intervention, and control measures are required to control the spread of MRSA among veterinarians.

\section{Acknowledgments}

We would like to thank the organizers of the different conferences and the responsible person at the Copenhagen Business School for allowing us to conduct this study.

The study was supported by the EU Marie Early Stage Training Programme TRAINAU (contract MESTCT-2004-007819).

\section{References}

1. Cosgrove SE, Sakoulas G, Perencevich EN, Schwaber MJ, Karchmer AW, Carmeli Y. Comparison of mortality associated with methicillin-resistant and methicillin-susceptible Staphylococcus aureus bacteremia: a meta-analysis. Clin Infect Dis. 2003;36:53-9.

2. Seybold U, Kourbatova EV, Johnson JG, Halvosa SJ, Wang YF, King MD, et al. Emergence of community-associated methicillin-resistant Staphylococcus aureus USA300 genotype as a major cause of health care-associated blood stream infections. Clin Infect Dis. 2006;42:647-56.

3. Rubin RJ, Harrington CA, Poon A, Dietrich K, Greene JA, Moiduddin A. The economic impact of Staphylococcus aureus infection in New York City hospitals. Emerg Infect Dis. 1999;5:9-17.

4. Weese JS. Methicillin-resistant Staphylococcus aureus in horses and horse personnel. Vet Clin North Am Equine Pract. 2004;20:601-13.

5. Lee JH. Methicillin (Oxacillin)-resistant Staphylococcus aureus strains isolated from major food animals and their potential transmission to humans. Appl Environ Microbiol. 2003;69:6489-94.

6. Leonard FC, Markey BK. Methicillin-resistant Staphylococcus aureus in animals: a review. Vet J. 2007, Epub 2007 Jan 9. doi:10.1016/j.tvj1.2006.11.008.

7. Voss A, Loeffen F, Bakker J, Klaassen C, Wulf M. Methicillinresistant Staphylococcus aureus in pig farming. Emerg Infect Dis. 2005;11:1965-6.

8. Scott GM, Thomson R, Malone-Lee J, Ridgway GL. Cross-infection between animals and man: possible feline transmission of Staphylococcus aureus infection in humans. J Hosp Infect. 1988;12:29-34.

9. Cefai C, Ashurst S, Owens C. Human carriage of methicillinresistant Staphylococcus aureus linked with pet dog. Lancet. 1994;344:539-40.

10. Loeffler A, Boag AK, Sung J, Lindsay JA, Guardabassi L, Dalsgaard A, et al. Prevalence of methicillin-resistant Staphylococcus aureus among staff and pets in a small animal referral hospital in the UK. J Antimicrob Chemother. 2005;56:692-7.

11. Weese JS, Caldwell F, Willey BM, Kreiswirth BN, McGeer A, Rousseau J, et al. An outbreak of methicillin-resistant Staphylococcus aureus skin infections resulting from horse to human transmission in a veterinary hospital. Vet Microbiol. 2006;114:160-4.

12. Moodley A, Stegger M, Bagcigil AF, Baptiste KE, Loeffler A, Lloyd DH, et al. spa typing of methicillin-resistant Staphylococcus aureus isolated from domestic animals and veterinary staff in the UK and Ireland. J Antimicrob Chemother. 2006;58:1118-23.

13. Hanselman BA, Kruth SA, Rousseau J, Low DE, Willey BM, McGeer A, et al. Methicillin-resistant Staphylococcus aureus colonization in veterinary personnel. Emerg Infect Dis. 2006;12:1933-8.

14. Wulf MWH, Sørum M, van Nes A, Skov R, Melchers W, Klaassen C et al. Prevalence of methicillin resistant Staphylococcus aureus in veterinarians: an international study. Clin Microbiol Infect. Epub 2007 Nov 7. doi: 10.1111/j.14690691.2007.01873 (http://www.blackwell-synergy.com/doi/ pdf/10.1111/j.1469-0691.2007.01873.x)

15. Wulf M, van Nes A, Eikelenboom-Boskamp A, de Vries J, Melchers W, Klaassen C, et al. Methicillin-resistant Staphylococcus aureus in veterinary doctors and students, the Netherlands. Emerg Infect Dis. 2006;12:1939-41. 
16. Wertheim HF, Vos MC, Boelens HA, Voss A, Vandenbroucke-Grauls CM, Meester MH, et al. Low prevalence of methicillin-resistant Staphylococcus aureus (MRSA) at hospital admission in the Netherlands: the value of search and destroy and restrictive antibiotic use. J Hosp Infect. 2004;56:321-5.

17. Kuehnert MJ, Kruszon-Moran D, Hill HA, McQuillan G, McAllister SK, Fosheim G, et al. Prevalence of Staphylococcus aureus nasal colonization in the United States, 2001-2002. J Infect Dis. 2006;193:172-9.

18. European Antimicrobial Resistance Surveillance System (EARSS). EARSS annual report 2005. Bilthoven, The Netherlands: EARSS Management Team, members of the Advisory Board, and national representatives of EARSS; c1995-2007 [Cited 05 December 2007].Available from: http://www.rivm. nl/earss/Images/EARSS\%202005_tcm61-34899.pdf.

19. Guardabassi L, Stegger M, Skov R. Retrospective detection of methicillin resistant and susceptible Staphylococcus aureus ST398 in Danish slaughter pigs. Vet Microbiol. 2007;122:3846.

20. Jonas D, Speck M, Daschner FD, Grundmann H. Rapid PCRbased identification of methicillin-resistant Staphylococcus aureus from screening swabs. J Clin Microbiol. 2002;40:18213.

21. Harmsen D, Claus H, Witte W, Rothgänger J, Claus H, Turnwald D, et al. Typing of methicillin-resistant Staphylococcus aureus in a university hospital setting by using novel software for spa repeat determination and database management. J Clin Microbiol. 2003;41:5442-8.

22. Oliveira DC, de Lencastre H. Multiplex PCR strategy for rapid identification of structural types and variants of the mec element in methicillin-resistant Staphylococcus aureus. Antimicrob Agents Chemother. 2002;46:2155-61

23. Lina G, Piemont Y, Godail-Gamot F, Bes M, Peter MO, Gauduchon V, et al. Involvement of Panton-Valentine leukocidin-producing Staphylococcus aureus in primary skin infections and pneumonia. Clin Infect Dis. 1999;29:1128-32.

24. Clinical and Laboratory Standards Institute (CLSI). Perfor- mance standards for antimicrobial disk susceptibility tests: approved standard. 9th ed. Wayne (PA): CLSI; 2006.

25. Toma E, Barriault D. Antimicrobial activity of fusidic acid and disk diffusion susceptibility testing criteria from Gram positive cocci. J Clin Microbiol. 1995;33:1712-5.

26. Fuchs PC, Jones RN, Barry AL. Interpretative criteria for disk diffusion susceptibility testing of mupirocin, a topical antibiotic. J Clin Microbiol. 1990;28,608-9.

27. Metha M, Patel N. LogXact 4 for windows: users manual. Cambridge (MA): Cytel Software Corporation; 2001.

28. de Neeling AJ, van den Broek MJ, Spalburg EC, van SantenVerheuvel MG, Dam-Deisz WD, Boshuizen HC, et al. High prevalence of methicillin resistant Staphylococcus aureus in pigs. Vet Microbiol. 2007;122:366-72.

29. Bagcigil FA, Moodley A, Baptiste KE, Jensen VF, Guardabassi $\mathrm{L}$, et al. Occurrence, species distribution, antimicrobial resistance and clonality of methicillin- and erythromycin-resistant staphylococci in the nasal cavity of domestic animals. Vet Microbiol. 2007;121:307-15.

30. Weese JS, Archambault M, Willey BM, Hearn P, Kreiswirth BN, Said-Salim B, et al. Methicillin-resistant Staphylococcus aureus in horses and horse personnel, 2000-2002. Emerg Infect Dis. 2005;11:430-5.

31. Baptiste KE, Williams K, Willams NJ, Wattret A, Clegg PD, Dawson S, et al. Methicillin-resistant staphylococci in companion animals. Emerg Infect Dis. 2005;11:1942-4.

32. Huijsdens XW, van Dijke BJ, Spalburg E, van Santen-Verheuvel MG, Heck ME, et al. Community-acquired MRSA and pig-farming. Ann Clin Microbiol Antimicrob. 2006;5:26.

33. Witte W, Strommenger B, Stanek C, Cuny C. Methicillin-resistant Staphylococcus aureus ST398 in humans and animals, Central Europe. Emerg Infect Dis. 2007; 13:255-8.

34. Weese JS, Caldwell F, Willey BM, Kreiswirth BN, McGeer A, Rousseau J, Low DE. An outbreak of methicillin-resistant Staphylococcus aureus skin infections resulting from horse to human transmission in a veterinary hospital. Vet Microbiol. 2006; 114:160-4. 35. 